\title{
The Factors Affecting the Increase in Overweight and Obesity on the Students of the University of Vlora (2012 - 2013)
}

\author{
Yllka Bilushi \\ Department of Health Sciences, Vlora University, Albania \\ Research Institute of Science \& Technology, United States
}

\section{Christos Kokaliaris}

chrisk.msor@gmail.com

\section{Doi:10.5901/mjss.2014.v5n27p1308}

\begin{abstract}
Overweight and Obesity share a common character that the disease is spreading in young people throughout the world. Today in the USA 1 in 4 children is overweight [1] and 65\% of the American people are considered obese [2], these reasons are more than enough to name the phenomenon "globesyity". The purpose of this study is the identification of the modifiable factors that affect the risk of overweight and obesity. The sample included 500 students, 323 of them were females and 177 males. The results from the research show that, the changes are considered statistically significant with a $p$-value of $p<0.05$. The factors that contribute on the BMI change among the students are related to: parents education, economic level of the family, the historic of health problems, the use of food that have a high level of proteins, sugars and fats and medicament usage.
\end{abstract}

Keywords: obesity, young people, overweight, BMI.

\section{Introduction}

The increase in the number of young people suffering from overweight and obesity is being observed more and more, in the current conditions of socio-economic development, with the increase of prosperity and the introduction of new technologies in our everyday life. This is noticeable in our country as well, with the change of the economical and social system. Before the years 1990 underweight was predominant in Albania.

In the developed countries such as the USA and several European countries the phenomenon is even more noticeable, the increase in the percentage of people suffering from overweight and obesity especially in the schooling age, it actually consists a problem to the social health. In the United States it is estimated that one in four children is overweight. [1] About $65 \%$ of the American citizens are considered to be overweight or obese. [2] According to the researchers, children in Greece suffer from obesity the most in comparison to other European countries.

It is therefore reasonable to ask ourselves the simple question: What are the reasons causing high levels of obesity? There are in fact several reasons that are responsible for this outcome. We begin with a reason that nobody can "condemn" : the endocrine irregularities and genetic inheritance, however the problem does not end there.

1. Dietary habits : Nutrition per capita and the required energy supplies vary markedly in different regions of the world. This however has changed significantly over time. [3] From the year 1970 until the early 1990s the average amount of calories available per person per day (the amount of food bought) was increased in all the parts of the world except Eastern Europe. The consumption of sweetened drinks is believed to have contributed to the increased rates of obesity. [4] The intake of more calories than the body requires leads to an increase in body fat. For every 7500 calories our body absorbs, our body gains about $1 \mathrm{~kg}$ of body fat, this leads to obesity. The increased number of fast food and sweets shops is also a noticeable factor.

2. Sedentary life: Throughout the globe there has been noticed a considerable decrease in the amount of physical activity [5], [6], [7] and nowadays at least $60 \%$ of the general population is considered to be physically inactive. This is mostly due to the increasing usage of the mechanized transportation and a greater prevalence of the mechanized labor. The spread of new technological devices related to housework seems also to play a role in this case. [5], [6], [7] In children there seems to be a decrease in the physical activity due to the fact that they seem to walk less. [8] There exists a relation between the time spent by watching the television and the risk of obesity among children and adults. [8] 
3. Psychic disease : Depression and stress lead to obesity and are among the main causes of dietary irregularities. Overweight people suffer from psychological problems more than people of normal weight. Several physical and mental illnesses and the pharmaceutic substances that have been used in order to prevent them may increase the risk of obesity.

4. Social factors : It is believed that developed and wealthy countries, are capable of covering the necessary costs in order to establish specific centers of rehabilitation for obese people. In the lesser developed countries the ability to cover the same expenses is way more limited in comparison to the developed countries, therefore it is usually required from the patients to spend more energy in the form of outdoors physical activities in order to decrease their excessive weight. [9]

5. Infectious agents : The study of the effects cause by infectious agents in the metabolism is still in early an early phase. A specific type of viruses that affect obesity has been found in humans and animals. These viruses might have contributed in the increase in the norm of obesity however it is still early for a definite answer. [10]

\section{The Common Consequences of Overweight and Obesity in Health}

Overweight and obesity can lead to serious health issues. The risk of chronic disease increases progressively with the increase in BMI. We should mention here the cardiovascular diseases, which now are the main cause of death to people worldwide, by killing approximately 17 million people a year. Diabetes is also noticeable and it can now be considered as a global epidemic. From the studies, is believed that death by diabetes will increase by more than $50 \%$ of cases worldwide in the upcoming 10 years. The list goes on with disease such as muscle - skeletal irregularities, especially osteoarthritis and several types of cancers ( endometrial, breast, colon). Obesity in children is connected to a higher chance of early death and disability when they become of age.

For several years a group of professors and students of the University of Vlora, had been studying the factors that affect the prevalence of overweight and obesity, to the students of the same university. The goal of their study was the identification of the modifiable factors regarding the risk of overweight and obesity in order to intervene effectively and prevent this problematic pathology, especially for the population under study.

\section{Objectives}

1. Gathering the statistical data of the indicators of the body mass of the students.

2. Identification of the deviation regarding the increase or decrease in the indicators of the body mass.

3. The prevalence of obesity between females and males.

4. The study of several factors that affect the increase of overweight and obesity such as: the economical, education level of the parents and disease suffered in childhood.

\section{Sample}

- The sample consists of students from the University of Vlora (2012 - 2013). This choice was made on purpose so that the sampling population was appropriate in order for the realization of the objectives of the study to be accomplished.

- The individuals cooperated well and were disposable on their on free will.

- Among the inclusive criteria, there was the year of study 2012 - 2013. The university disposes of about four thousand students that originate from several geographic locations. In our study through questionnaire there were included in total 500 students that are divided in two categories depending on gender, 323 females and 177 males.

- From this study there were excluded the boarding students, since their diet is different than the population we are taking under study.

- There has also been excluded the cases where the students suffered from chronic disease, so that the study could be generalized with regards to the healthy majority. 


\section{Methodology}

The gathering of data was carried in two phases:

- The first phase consisted of answering to the questionnaire. From these students was filled a detailed questionnaire, which aimed at extracting the significant data that define the BMI.

- The second phase consisted on the performing of the anthropometric measurements. All the above students underwent the anthropometric measurements of weight and length parameters.

The students were chosen randomly from a trained student who was being accompanied by a professor of the university. The participation of the students was quite high and the students who agreed to participate in the study were asked to sign their consent in accordance to the Helsinki standards.

The questionnaire provides us with information regarding the contributing factors on the change of body mass to the students in conjunction with:

- The education level of their parents

- The economical status of their family

- The historic of their health problems, that affect in the increase or decrease of the body mass index.

- The consumption of foods that consist of a high amount of carbohydrates, proteins and fats.

- The consumption of fruit in everyday nutrition.

- The consumption of medicament.

- The consumption of alcoholic beverages and soft drinks containing sugar.

\section{Data Analysis}

1. The data analysis was carried by utilizing the statistical software SPSS 13.0

2. The analysis of variance was performed for the examination of the characteristic anthropometric differences between the students. The results were expressed in the form of $\pm s d$ ( standard deviation). The changeable parameters were analyzed by applying t-test to the students, while the corresponding results were utilized in nonparametric inference.

3. All the reported $p$-values were based on the two tailed test with a statistical significance $a=5 \%$. The BMI was calculated through the statistical software SPSS v13.0. The questionnaire used was adopted by a previously published article, [12] which was standardized for the age groups under study. Before filling the questionnaire the students were informed about the study. They were given instructions on how to properly complete the questionnaire. The physical measurements of weight and height took place before filling the questionnaire.

4. The percentages of bodily indicators were established using electronic scales. The fluctuations regarding the status of bodily hydration might affect in the obtained results, therefore the conditions for the weighting were that it should take place in morning hours, at least three hours after wakeup where the students physique was empty, they hadn't done any workout and hadn't eaten or drank at least 3 hour before the weighting.

5. All students underwent the process of cleansing the soles of their feet before they could step on the scale in order to avoid fluctuations in the measurements. The height was measured using a metallic ruler. The students were requested to remove their shoes for the height measurement.

\section{The Results}

There were 500 students participating and we divided them according to their gender where 323 are females and 177 are males. Based on their gender we classified them with regards to their BMI.

Table 1: BMI in accordance to gender.

\begin{tabular}{|c|c|c|}
\hline BMI & Female & Male \\
\hline Underweight & 84 & 9 \\
\hline Normal & 214 & 109 \\
\hline Overweight & 23 & 53 \\
\hline Obese & 2 & 6 \\
\hline
\end{tabular}


Following up we present several graphical representations on how the BMI varies depending on some of the factors under study.

BMI for females/males in accordance to parents education

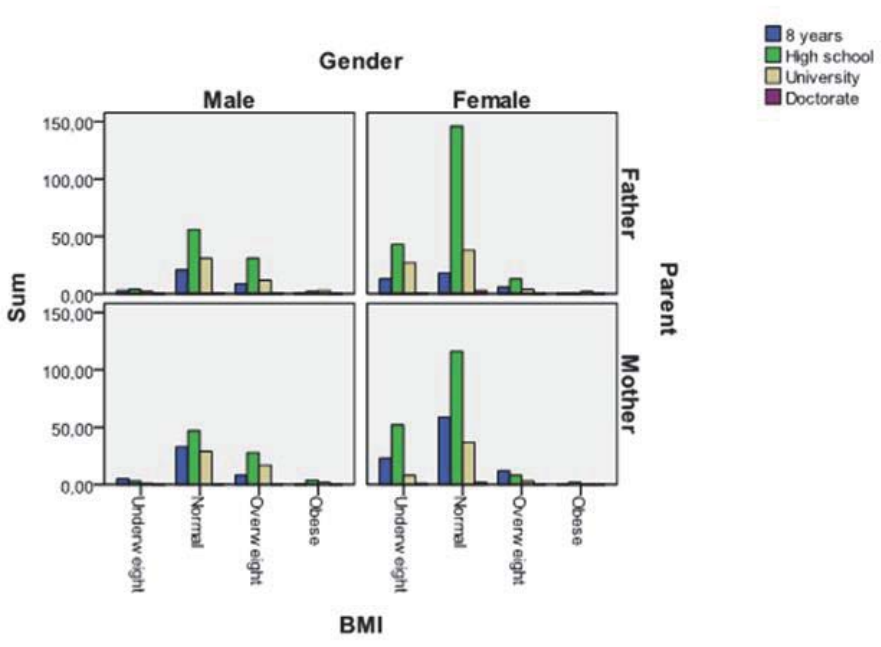

BMI for females/males in accordance to working family members

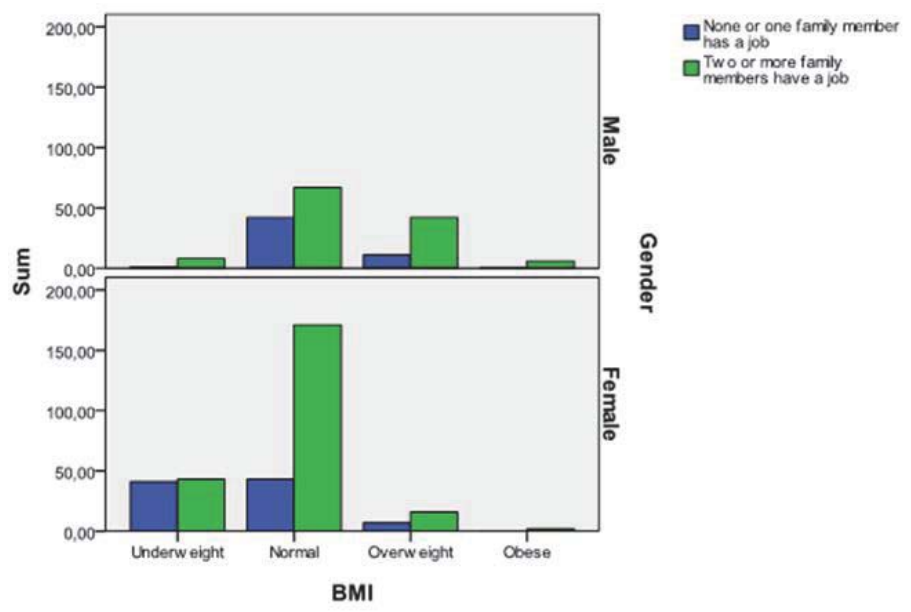

BMI for females/males in accordance to fruits and medication

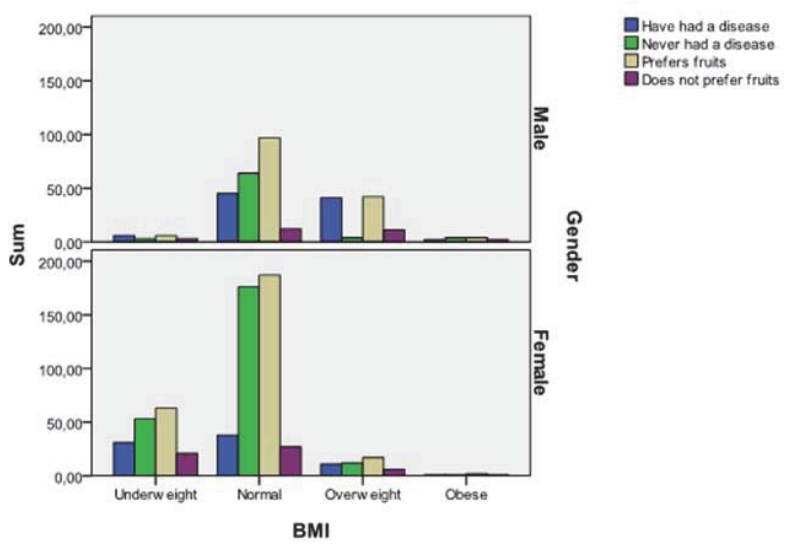




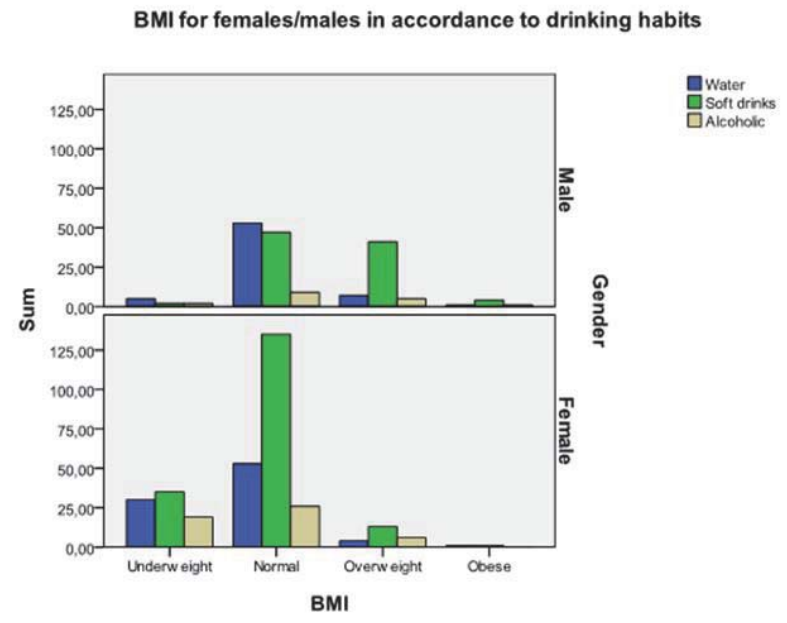

BMI for females/males in accordance to prtoteins, carbs, fats consumption

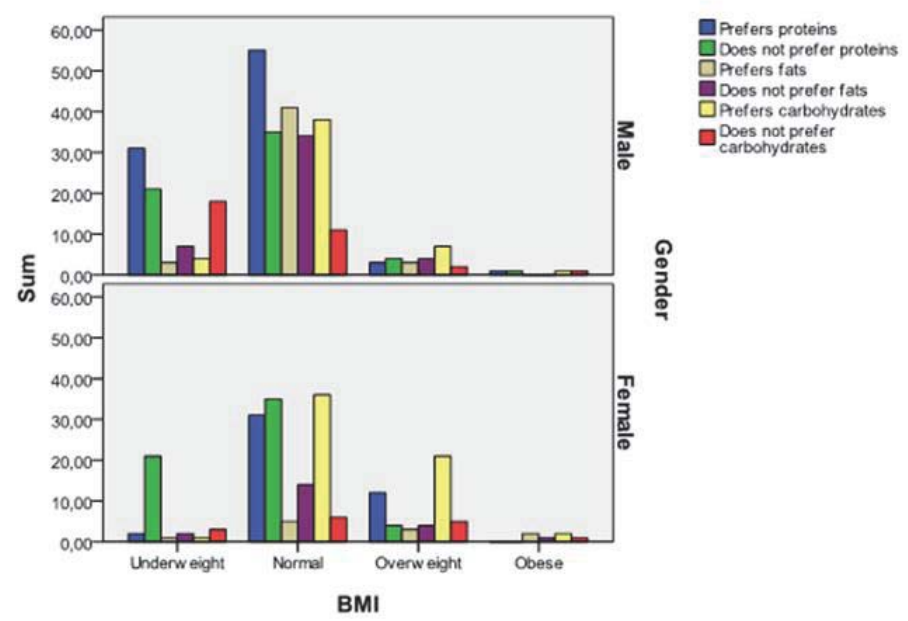

\section{Statistically Significant Factors}

1. We compare the females and the males in every weight category regarding the BMI. The Pearson chi-squared test with 3 degrees of freedom resulted statistically significant with a $p$-value $=0.000$. This resulted that males tend to show higher levels of overweight and obesity in comparison to females.

2. Comparing the level of mother's education to the BMI in males the test results statistically significant with a pvalue $=0.0312$. On average we notice a tendency of higher BMI levels of overweight and obesity on males when the mother's education is high-school or higher.

3. Comparing the BMI to the number of family members working we obtain a $p$-value $=0.0191$ for the males and $p$-value $=0.000$ which is statistically significant. This practically means that males or females who have at least two working family members show a tendency towards higher levels of obesity and overweight.

4. The test between BMI and past disease proves statistically significant with a p-value $=0.000$ for both males and females. It results that on average students either males or females who have suffered from past diseases show a lower tendency towards obesity and overweight in comparison to students who have never suffered from a disease in the past.

5. For the females the test between BMI and carbohydrates consumption is statistically significant with a p-value $=0.038$, meaning that there is a tendency that higher preference on carbohydrates might lead to a higher level of overweight or obesity.

6. For the females the test between BMI and fruits consumption is statistically significant with a $p$-value $=0.0213$, meaning that an increase in fruits consumption leads to a tendency in increased BMI. For the males the $p$ - 
value $=0.0544$ meant the test was not statistically significant.

7. Regarding the medicament consumption and BMI levels on the females the $p$-value $=0.000$ means there is a statistically significant relation and the medicaments that females use can lead to increased levels of BMI. This was not the case with males where the $p$-value $=0.4695$ means the test is statistically insignificant.

8. Regarding the consumption of alcoholic beverages or soft drink and their relation to the BMI the Pearson chisquared test with 3 degrees of freedom was statistically significant with $p$-value $=0.000$ for both males and females. The consumption of alcoholic beverages or soft drinks it appears to lead to increased levels of BMI.

\section{Final Results and Recommendations}

The prevention and treatment of overweight and obesity will aid us towards a healthier generation.

Overweight is a risk factor for many pathologies, while obesity is a complex, longterm disease. While being as such their treatment is difficult and tiresome to the employees of the health service (medics, nurses, psychologists) as is to the patients themselves. The treatment of overweight and obese children and teenagers have brought us longterm benefits. Several programs have lead to weight stability for the patients up to ten years, [2] this resulted to $30 \%$ of the students that participated in this program, although some of the children still remained overweight in a lower percentage. These programs include interventions to reduce the calories intake and the increase of the physical activity level. Specialists in the treatment of overweight and obesity have identified and isolated several components for a successful treatment involving:

1. The treatment must start before the age of adolescence if possible.

2. The students themselves must be consciously willing to participate.

3. The family is informed about the complications cause by overweight and obesity.

4. The entire family is involved during the treatment.

5. The longterm decrease in weight is more advisable than the sudden decrease in a short amount of time. The changes must happen in a gradual manner.

6. There are recommended activities that contribute to keeping under control the dietary habits and the physical activities.

These are the key points of the program that must be followed by the patient in order to achieve the desired results within the community that he might belong. [4] It should be brought under consideration the necessity to create an arsenal of knowledge and strategies in the service of all students population.

- It is worth mentioning that the feeding process is the total resultant of the interaction between the organism and the food that it consumes.

- Young people need to be informed continuously regarding their diet, what they should consume and what they shouldn't. Each individual is responsible for the choice of food that ensures him an appropriate diet.

- The assistance of people in order to be able to assess and take into account the information they receive about food. The essential foods are grouped into six categories water, carbohydrates, fats, proteins, vitamins, minerals.

- It should be noted that the nutrients serve for three main purposes:

- Formation of body structures such as bones and blood. [6]

- Energy source.

- Help the body to regulate the biochemical reactions.

- The side effects of malnutrition might be general or specific which vary due to the nutrients and their lack or abundance levels.

- It is also noteworthy to mention some of the side effects caused as a result of high consumption of food, here we include several diseases as cancer, coronary artery disease, second type diabetes and liver disease. [8] The nutritional needs depend on age, growth, energy demands ect. Teenagers need more energy, this due to their rapid development. A diet consisting of milk, meat, vegetables and fresh fruit is ideal in this case. [9]

- In order to asses the physical development of an individual the knowledge of the following values is required [10]:

- Anthropometric measurements, length, weight, abdominal and groin circumferences.

- The biochemical analyses, total cholesterol, LDL, HDL, triglycerides.

- Clinical signs associated with the level of nutrition. 
- A dietary historic

- When we advise the young people in order to achieve the optimal nutritional goals, we should take under account the factors that influence a person's diet.

\section{References}

Calle EE, Rodriguez C, Walker-Thurmond K, Thun MJ (April 2003). "Overweight, obesity, and mortality from cancer in a prospectively studied cohort of U.S. adults". N. Engl. J. Med. 348 (17): 1625-38.doi:10.1056/NEJMoa021423. PMID 12711737.

Poulain M, Doucet M, Major GC, et al. (April 2006). "The effect of obesity on chronic respiratory diseases: pathophysiology and therapeutic strategies". CMAJ 174 (9): 1293-9. doi:10.1503/cmaj.051299.PMID 16636330. PMC 1435949

Caballero B (March 2001). "Introduction. Symposium: Obesity in developing countries: biological and ecological factors". J. Nutr. 131 (3): 866S-870S. PMID 11238776.

Tate DF, Jeffery RW, Sherwood NE, Wing RR (01 April 2007). "Long-term weight losses associated with prescription of higher physical activity goals. Are higher levels of physical activity protective against weight regain?". Am. J. Clin. Nutr. 85 (4): 954-9. PMID 17413092

Norris SL, Zhang X, Avenell A, Gregg E, Schmid CH, Lau J (2005). "Pharmacotherapy for weight loss in adults with type 2 diabetes mellitus". Cochrane database of systematic reviews (Online) (1): CD004096.doi:10.1002/14651858.CD004096.pub 2. PMID 15674929..

Healthy Weight: Assessing Your Weight: BMI: About BMI for Children and Teens" (html). Center for disease control and prevention. Retrieved April 6, 2009.

Neumark-Sztainer D (March 1999). "The weight dilemma: a range of philosophical perspectives". Int. J. Obes. Relat. Metab. Disord. 23 Suppl 2: S31-7. doi:10.1038/sj.ijo.0800857. PMID 10340803. 92.

Dollman J, Norton K, Norton L (December 2005). "Evidence for secular trends in children's physical activity behaviour". Br J Sports Med 39 (12): 892-7; discussion 897. doi:10.1136/bjsm.2004.016675. PMID16306494

^ Christakis NA, Fowler JH (2007). "The Spread of Obesity in a Large Social Network over 32 Years". New England Journal of Medicine 357 (4): 370-379. doi:10.1056/NEJMsa066082. PMID 17652652

^ Falagas ME, Kompoti M (July 2006). "Obesity and infection". Lancet Infect Dis 6 (7): 438-46. doi:10.1016/S1473-3099(06)70523-0. PMID 16790384.

Obesity and the facts, An analysis of data from the Health Survey for England Conducted by the Social Issues Research Centre,February 2003.

SAD Eknoyan, Garabed (January 2008). "Adolphe Quetelet (1796-1874)-the average man and indices of obesity". Nephrol. Dial. Transplant. 23 (1): 47-51. doi:10.1093/ndt/gfm517. PMID 1789075 